Three Minute Article for Parents

\title{
How far could the mobile applications aid in maintaining maternal and child healthcare in times of COVID-19 pandemic?
}

\author{
Shreyasi Roy iD https://orcid.org/0000-0002-6024-052X
}

Sri Lanka Journal of Child Health, 2022: 51(1): 168

DOI: http://dx.doi.org/10.4038/sljch.v51i1.10035

(Keywords: Mobile applications, Digital world)

Corona virus disease in 2019, better known as COVID-19, has now become a serious pandemic worldwide after its initial outbreak in Wuhan, China in late 2019. During its second wave (and subsequent shorter waves), India has observed a massive COVID surge which has inflicted a major impact on the healthcare system. At such crucial times, it becomes critical to maintain preventive and curative health services, especially for the vulnerable group of populations including children, pregnant women and lactating mothers.

Since the utmost precaution one could adopt during this pandemic is social distancing, the virtual world has become the norm. As such, online classes, online meetings and conferences, digital mode of payment, etc. are adopted by people worldwide. Healthcare systems have embraced a shift to the virtual world for a long time. Mobile phone applications launched by government, private, several national and international institutes have aimed at reducing maternal and child health issues in India ${ }^{1}$.

Some of the renowned applications launched in India were Mobile for mothers (Mfm), mobile Mitra (mMitra), Khushi baby, ShishuPoshan and Kilkari. Most of these services were confined to a particular state or a specific location. However, there are several applications available in Google Play Store which could be accessed by expectant mothers / lactating mothers / new mothers at any place. Examples of such applications include MomJunction - Your Pregnancy Guide, Parentlane, Parentune - Parenting, Child care Growth Tracker, Babygogo Parenting - Baby Care \& Pregnancy Tips, Mylo: Pregnancy and Baby Companion, Healofy: Indian pregnancy, Parenting Tips \& baby Products, Happy Pregnancy App. These applications have received several positive reviews worldwide in yielding scientific and useful information on topics related to pregnancy, breastfeeding, child care and parenting, most of which comes in illustrative pictures and videos.

Some of these applications also provide a platform where a user (pregnant/ lactating / new mothers / others) gets connected to several fellow users and can have access to several queries. In some of these applications, they often get vetted by experts, doctors and nutritionists. In a nutshell, they can contribute as a great health guide at any place and at any time.

The take-home message is that all expectant and/or new mothers should embrace the digital world in terms of health care management. This would possibly prove to be beneficial in times of formidable threats such as COVID-19.

\section{References}

1. Roy S, Sen J. Maternal and Child Health Care in the Era of Mobile Applications. In: Mondal N, Singh KN. Maternal Health and Child Mortality: Condition, Vision and Challenges. Concept Publishing Company, New Delhi; 2021: 345-359. 